\title{
Virus-induced plasma membrane aquaporin PsPIP2;1 silencing inhibits plant water transport of Pisum sativum
}

\author{
Juanjuan Song ${ }^{1,2}$, Guoliang Ye $e^{1,3}$, Zhengjiang Qian ${ }^{1,3}$ and Qing Ye $e^{1,2^{*}}$
}

\begin{abstract}
Background: Aquaporins (AQPs) are known to facilitate water transport across cell membranes, but the role of a single AQP in regulating plant water transport, particularly in plants other than Arabidopsis remains largely unexplored. In the present study, a virus-induced gene silencing (VIGS) technique was employed to suppress the expression of a specific plasma membrane aquaporin PsPIP2;1 of Pea plants (Pisum sativum), and subsequent effects of the gene suppression on root hydraulic conductivity $\left(L_{\mathrm{r}}\right)$, leaf hydraulic conductivity $\left(K_{\text {leaf }}\right)$, root cell hydraulic conductivity $\left(\mathrm{LP}_{\mathrm{rc}}\right)$, and leaf cell hydraulic conductivity $\left(L p_{\mid c}\right)$ were investigated, using hydroponically grown Pea plants.

Results: Compared with control plants, VIGS-PsPIP2;1 plants displayed a significant suppression of PSPIP2;1 in both roots and leaves, while the expression of other four PIP isoforms (PSPIP1;1, PSPIP1;2, PsPIP2;2, and PSPIP2;3) that were simultaneously monitored were not altered. As a consequence, significant declines in water transport of VIGS-PsPIP2;1 plants were observed at both organ and cell levels, i.e., as compared to control plants, $L \mathrm{P}_{\mathrm{r}}$ and $K_{\text {leaf }}$ were reduced by $29 \%$, and $L p_{\mathrm{rc}}$ and $L \mathrm{p}_{\mathrm{lc}}$ were reduced by 20 and $29 \%$, respectively.

Conclusion: Our results demonstrate that PSPIP2;1 alone contributes substantially to root and leaf water transport in Pea plants, and highlight VIGS a useful tool for investigating the role of a single AQP in regulating plant water transport.
\end{abstract}

Keywords: Cell pressure probe, Hydraulic conductivity, Plant water relations, VIGS, Water channels

\section{Background}

Plant water relations are continually challenged by diverse environmental stimuli, such as light, temperature, soil water availability, and atmospheric humidity. To keep water homeostasis, plants need to respond promptly to the ever-changing environments via regulating water transport at cellular, tissue, organ, and whole plant level (Aroca et al. 2012; Bramley et al. 2009; Chaumont and Tyerman 2014; Chevalier and Chaumont 2015; Henry et al. 2012; Luu and Maurel 2005). Aquaporins (AQPs) are trans-membrane proteins that facilitate rapid and

\footnotetext{
*Correspondence: qye@scbg.ac.cn

${ }^{1}$ Key Laboratory of Vegetation Restoration and Management

of Degraded Ecosystems, South China Botanical Garden,

Chinese Academy of Sciences, 723 Xingke Road, Tianhe District,

Guangzhou 510650, China

Full list of author information is available at the end of the article
}

passive water transport across cell membranes. According to sequence homology and sub-cellular localizations, plants AQPs can be classified into seven subfamilies, i.e., plasma membrane intrinsic proteins (PIPs), tonoplast intrinsic protein (TIP), NOD26-like intrinsic proteins (NIPs), small intrinsic proteins (SIPs), X-intrinsic proteins (XIPs), GlypF-like intrinsic proteins (GIPs), and hybrid intrinsic proteins (HIPs) (Anderberg et al. 2011; Gustavsson et al. 2005; Johanson et al. 2001; Li et al. 2014). Among them, PIPs constitute the largest number and can be further divided into two subgroups named PIP1 and PIP2 (Ayadi et al. 2011; Chaumont et al. 2000; Johansson et al. 2000).

The role of AQPs in regulating plant water transport has been abundantly documented, and PIPs represent the most likely candidates for protein-mediated hydraulic conductivity in plants (Heinen et al. 2009; Maurel et al. 
2008). The contribution of AQPs to plant hydraulic conductivity has been tested by variable approaches. The first notion that AQPs involving in plant water transport was raised from experiments showing that root water transport can be substantially inhibited by AQP blocker, i.e., mercurial regents (Javot and Maurel 2002; Maggio and Joly 1995; Zhang and Tyerman 1999). Because mercury compound showed inhibitive effects in general on other physiological processes besides blocking AQPs, more specific approaches involved the use of transgenic plants with altered expression of targeted PIPs were employed (Jang et al. 2007; Javot et al. 2003; Lee et al. 2012; Postaire et al. 2010; Secchi and Zwieniecki 2014; Yu et al. 2005). For instance, over-expression of Arabidopsis PIP1b in tobacco improved plant vigor under favorable growth condition (Aharon et al. 2003). Low temperature induced reductions in cell hydraulic conductivity was alleviated by over-expressing AtPIP2;5 in Arabidopsis plants (Lee et al. 2012). In grapevine, it was found that the over-expression of a root specific AQP $V v P I P 2 ; 4 N$ enhanced water transport at the whole plant level (Perrone et al. 2012). By contrast, hydraulic conductivity of root cortex cell was reduced by $25-30 \%$ in PIP2;2 knockout mutant of Arabidopsis plants (Javot et al. 2003), and a reduction of about $20 \%$ in the relative water flux into rosette leaves was found in these mutants (Da Ines et al. 2010). Similarly, disruption of AtPIP1;2 resulted in a significant decrease (by 20-30\%) in root hydraulic conductivity of Arabidopsis (Postaire et al. 2010), while PIP1 and PIP2 double antisense Arabidopsis plants had a threefold decrease in the root hydraulic conductivity (Martre et al. 2002). All these pioneer findings pointed to the important roles of AQPs in regulating water transport across diverse species, while the contribution of a single AQP to hydraulic conductions in plants other than Arabidopsis remains to be explored.

Virus-induced gene silencing (VIGS) is a reverse genetics technology that can produce a rapid, sequencespecific knockdown phenotype for the target gene (Burch-Smith et al. 2004). To this end, a fragment of the target gene is inserted into a viral delivery vector which is used to infect plants. During the inoculation, virus replication triggers the natural defense mechanisms of plants to suppressing virus replication, which is also result in specific degradation of mRNAs from the endogenous gene that is targeted for silencing (Baulcombe 1999; $\mathrm{Lu}$ et al. 2003). Therefore, compared with other transgenic methods, VIGS technology represents a simple but attractive reverse-genetics tool for gene functional studies (Pflieger et al. 2013). In addition, VIGS does not need to develop stable transformants, thus can be used to study the function of genes that might be fatal for plants when such functions are impaired in stable transformed lines (Burch-Smith et al. 2004; Purkayastha and Dasgupta 2009). With these advantages, VIGS technology has been broadly applied for functional studies of specific genes across a number of plant species including Tobacco, Arabidopsis, Tomato, Rice, and Pea plants (Constantin et al. 2004; Fragkostefanakis et al. 2014; Purkayastha et al. 2010; Senthil-Kumar and Mysore 2014).

\section{Results and discussion}

In this study, five PIP isoforms (PsPIP1;1, PsPIP1;2, PsPIP2;1, PsPIP2;2, and PsPIP2;3) were identified and cloned in Pisum sativum. In the preliminary trials, our quantitative real-time PCR (q-RT-PCR) results revealed that the expression of PSPIP2;1 was the highest in roots among the three study PIP2s (Fig. 1). Along with previous findings that PSPIP2;1 showed marked water transport activity when expressed in Xenopus oocytes (Schuurmans et al. 2003), and its expression pattern displayed a tight correlation with the diurnal change in root hydraulic conductivity (Beaudette et al. 2007), we therefore chose PsPIP2; 1 as the primarily target gene to explore its contribution to water transport in Pea plants. Firstly, we employed the VIGS method to suppress the expression of PSPIP2;1, which was quantified using q-RT-PCR, with the expression of the other four PIPs being monitored simultaneously. Subsequently, changes in root and leaf hydraulic conductivities of VIGS-PsPIP2;1 plants at both organ and cell levels were determined using pressure chamber and cell pressure probing techniques, respectively, and the role of PSPIP2;1 in regulating Pea plant water transport was discussed.

There is increasing evidence supporting the crucial roles of AQPs in regulating plant water transport (Aroca et al. 2012; Chevalier and Chaumont 2015; Li et al. 2014). However, due to high multiplicity of plant AQP isoforms, the contribution of a specific AQP gene to hydraulic conductivity of non- Arabidopsis plants remains largely unknown. In the present study, five full-length cDNAs of plasma membrane aquaporins (PIPs) were isolated from Pea plants. Among them, two genes belong to the PIP1 subfamily, and the other three belong to the PIP2 subfamily, which were designated as PsPIP1;1, PsPIP1;2, PsPIP2;1, PSPIP2;2 and PsPIP2;3, respectively (Fig. 2). Taking the advantage of VIGS method, we intended to investigate the contribution of PSPIP2;1 to water transport in both roots and leaves of Pea plants. Because AQPs constitute a large and highly divergent protein family in plants, it is important to carefully analyze possible compensation effects by closely related isoforms when studying the function of a specific AQP through modifying its expression (Heinen et al. 2009). For example, the transcript levels of endogenous PIPs was noticeably affected by the over-expression of PIP1;4 and PIP2;5 


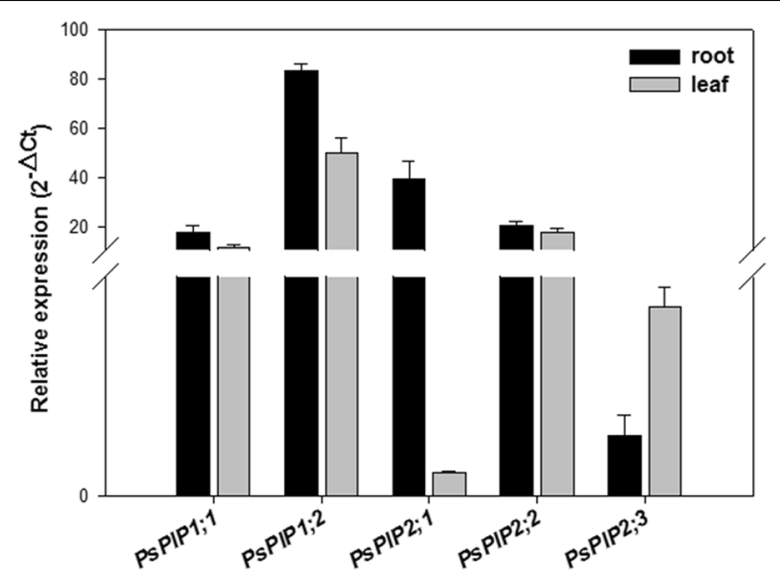

Fig. 1 Expression profiles of the five cloned PsPIPs in roots and leaves of Pea (Pisum sativum) plants

in Arabidopsis plants under water stress conditions, it is therefore difficult to attribute the observed phenotypes to the abundance change of target gene or to the altered expression of other endogenous AQPs (Jang et al. 2007). In the present study, our q-RT-RCR analysis confirmed that the mRNA expression of PSPIP2; 1 was significantly inhibited in both roots and leaves without altering the expression of the other four PIP isoforms (Fig. 3). Therefore, it might be reasonable to attribute changes in plant hydraulic conductivity to the suppression of PsPIP2;1, although alterations in the expression of other PIPs that are similar to PSPIP2;1 but not yet identified cannot be completely ruled out.

At the root level, a reduction of $L p_{r}$ by $29 \%$ was observed in VIGS-PsPIP2;1 plants compared with control plants (Fig. 4b). Cell volume (V), surface area (A), turgor pressure $(\mathrm{P})$, and elasticity $(\varepsilon)$ were not affected significantly by the virus-induced PsPIP2;1 silencing (Table 1 ), but the half-time of water exchange $\left(\mathrm{T}_{1 / 2}\right)$ increased from $1.8 \mathrm{~s} \mathrm{(on} \mathrm{average)} \mathrm{to} 2.6 \mathrm{~s}$ (Fig. 4c), resulting in a decrease of $\mathrm{Lp}_{\mathrm{rc}}$ by $20 \%$ in VIGS-PsPIP2;1 plants compared with the control plants (Fig. 4d). Our results are comparable to observations in Arabidopsis knockout mutants. On one hand, both the cell and the root hydraulic conductivity of Pea plants measured in this study were at the same magnitude as those of Arabidopsis plants (Javot et al. 2003; Postaire et al. 2010); on the other hand, a reduction of $20-30 \%$ in root hydraulic conductivity was observed in PIP1;2 mutants (Postaire et al. 2010), and hydraulic conductivity of root cortex cell was reduced by $25-30 \%$ in PIP2;2 mutant (Javot et al. 2003). These findings pointed to a substantial contribution of a single AQP isoform to root water transport. Along with the finding that the expression of PSPIP2;1 was positively correlated with the diurnal changes in root hydraulic conductivity of Pea plants (Beaudette et al. 2007), here our results provided further evidence that PSPIP2;1 was indeed involved in mediating root water transport in Pea plants.

The values of leaf hydraulic conductivities at both organ and cell levels that we measured in Pea plants were comparable to those of many other species such as Maize (Kim and Steudle 2007), Arabidopsis (Prado et al. 2013), and Cucumber (Qian et al. 2015). As found in roots, significant reductions in leaf hydraulic conductivities were observed when the expression of PsPIP2;1 was suppressed. For instance, $K_{\text {leaf }}$ were $183.3 \pm 24.1$ and $130.4 \pm 18.8 \mu \mathrm{L} \mathrm{s}^{-1} \mathrm{~m}^{-2} \mathrm{MPa}^{-1}$ in the control and VIGS-PsPIP2;1 plants, respectively, indicating that $K_{\text {leaf }}$ decreased by $29 \%$ (Fig. 5b). Without significant differences in cell geometry, turgor pressure, and cell wall elasticity between the control and VIGS-PsPIP2;1 plants (Table 1), we found that $\mathrm{T}_{1 / 2}$ increased (on average) from 1.2 to $1.7 \mathrm{~s}$ (Fig. 5c), indicating a decrease of leaf cell hydraulic conductivity by $29 \%$ in VIGS-PsPIP2;1 plants compared with the control plants (Fig. 5d). In leaves, it has been shown that alteration of AQP expression significantly affected leaf hydraulic conductivity, indicating the crucial roles of AQPs in plant leaf water transport (Cochard et al. 2007; Ding et al. 2004; Lopez-Berenguer et al. 2008; Muries et al. 2013). In the present study, we found the silencing of the PSPIP2; 1 resulted in a reduction of $29 \%$ the leaf hydraulic conductivities at both organ and cell levels, which was consistent with findings of previous studies. For instance, employing a deuterium tracer method, Da Ines et al. (2010) demonstrated that water flux into the Arabidopsis rosette was significantly reduced by about $20 \%$ inPIP2;1 and PIP2;2 knockout plants. By measuring Arabidopsis rosette water flux of three single PIP (PIP1;2, PIP2;1, or PIP2;6) knockout mutants, Prado et al. (2013) observed a significant reduction of water flux by $16-35 \%$ as compared to wild type, and the authors concluded that PIP1;2, PIP2;1, and PIP2;6 are important contributors of AQP-mediated rosette water transport. In this study, we noted that the expression of PSPIP2;1 in leaves was relatively low, still the reduction in leaf hydraulic conductivity of VIGS-PIP2;1 plants was significant. Therefore, except for expression level, the activity and/or localization of PSPIP2;1 might be critical in regulating Pea plant leaf water transport, as being pointed out by different researchers in a number of recent studies (Chevalier and Chaumont 2015; Kaneko et al. 2015), which deserve further investigations.

\section{Conclusions}

Our results demonstrated that the expression of PsPIP2;1 in Pea plants was specifically suppressed through the VIGS method. As a result, both root and leaf hydraulic conductivities were significantly reduced 


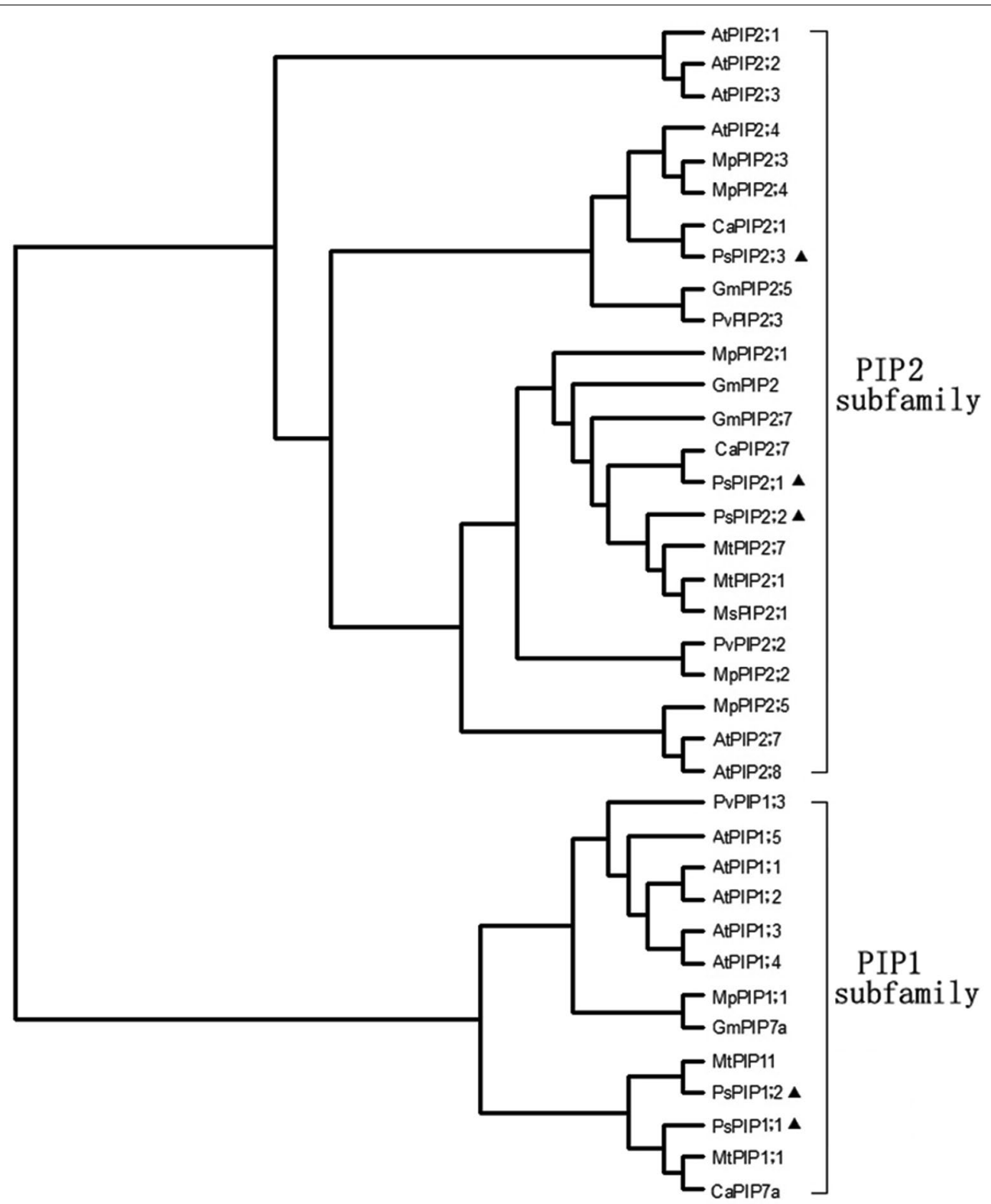

Fig. 2 Phylogenetic tree of AQPs from Pisum sativum sequences and other plants generated in MEG5.1 software. Subfamilies are labeled by brackets at the right side. Sequences of P. sativum are indicated by filled triangles. Information of the known 32 AQP sequences and GenBank accession numbers used are as follows: Arabidopsis thaliana, AtPIP1;1 (AEE80201), AtPIP1;2 (AEC10622), AtPIP1;3 (AEE27312), AtPIP1;4 (AEE81879), AtPIP1;5 (AEE84748), AtPIP2;1 (AEE79084), AtPIP2;2 (AEC09362), AtPIP2;3 (AEC09363), AtPIP2;4 (AED97364), AtPIP2;7 (AEE86464), AtPIP2;8 (AEC06543); Mimosa pudica, MpPIP1;1 (BAD90696), MpPIP2;1 (BAD90697), MpPIP2;2 (BAD90698), MpPIP2;3 (BAD90699), MpPIP2;4 (BAD90700), MpPIP2;5 (BAD90701); Cicer arietinum, CaPIP7a(XM_004490904), CaPIP2;1(XM_004496224), CaPIP2;7(XM_004505936); Glycine max, GmPIP2;7(XM_003538126), GmPIP2 (XM_003540128), GmPIP;7a (XM_003544062), GmPIP2;5 (XM_003556184); Medicago truncatula, MtPIP11(XM_003600815), MtPIP1;1(AF386739), MtPIP2; (AY059380), MtPIP2;7(XM_003606335); Phaseolus vulgaris, PvPIP1;3 (DQ855475), PvPIP2;2 (EF624001), PvPIP2;3 (EF624002); Medicago sativa subsp. Falcate, MsPIP2;1 (FJ607305); Pisum sativum, PsPIP1;1 (X54357), PsPIP1;2 (KF770828), PsPIP2;1 (AJ243307), PsPIP2;2 (KF770829), PsPIP2;3 (KF770830)

in PsPIP2;1-silenced plants compared with control plants. Consistent with previous findings that PsPIP2;1 showed marked water transport activity when expressed in Xenopus oocytes, and displayed a tight correlation with the diurnal change in root hydraulic conductivity, our results provided further evidence that PsPIP2;1 play an important role in regulating Pea plant water transport. However, precise mechanisms by which this AQP 


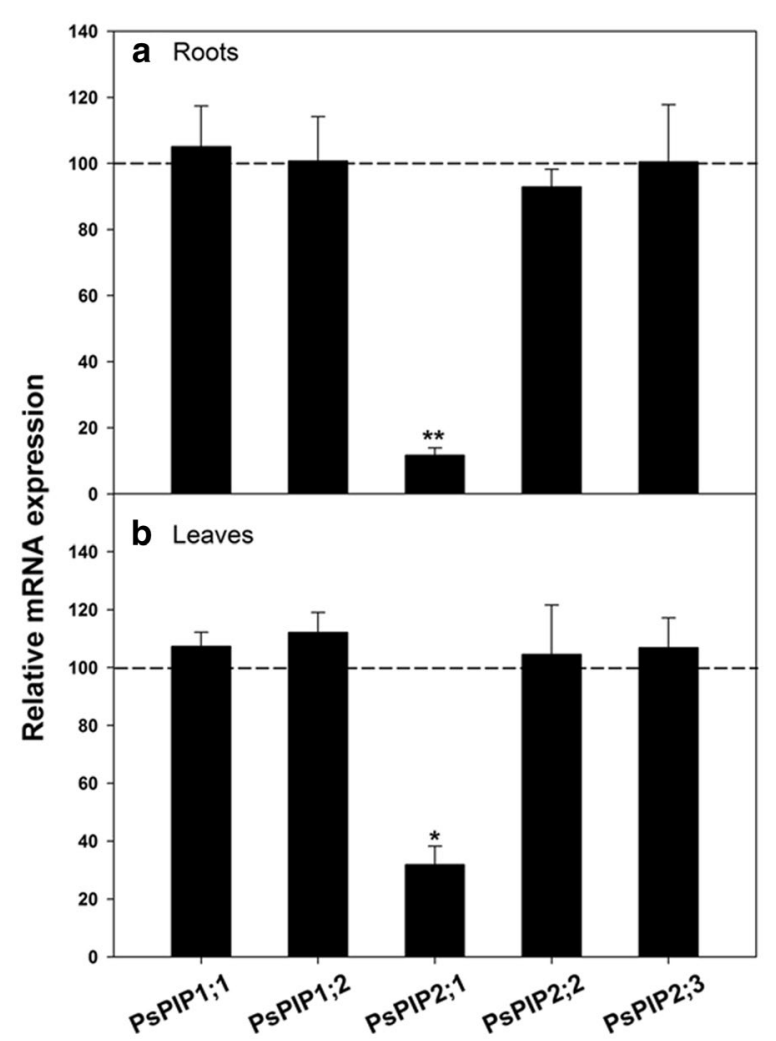

Fig. 3 Relative expression of PsPIP genes in roots ( $a$ ) and leaves ( $b$ ) of control plants and VIGS-PsPIP2;1 plants determined by quantitative real-time PCR. Values are mean \pm SD of three independent replicates. Asterisks or two asterisks ( ${ }^{*}$ or ${ }^{* *}$ ) represent a significant difference between VIGS-PsPIP2;1 plants and the control plants at $\mathrm{P}<0.05$ or $P<0.01$

mediates plant water transport remain to be explored. For instance, whether PSPIP2;1 had a tissue specific expression pattern in root endodermis and/or leaf bundle sheath that are proven to be critical in the pathway of plant water transport, as well as the responsiveness of PsPIP2;1 to abiotic stresses (e.g., drought stress), all merit future investigations.

\section{Methods}

\section{Plant material and growth conditions}

Pea plants (P. sativum L. line J1992) used in this study was obtained from National Key Laboratory of Plant Molecular Genetics, Institute of Plant Physiology and Ecology, Chinese Academy of Sciences, Shanghai, China. Seeds were germinated in wet filter paper in covered Petri dishes for 3 days at room temperature in the dark. Then seedlings were transferred to a hydroponic culture plastic box $(7 \mathrm{~L})$ filled with modified Hoagland solution $\left(\mathrm{pH}=6.0 ; 1.25 \mathrm{mM} \mathrm{Ca}\left(\mathrm{NO}_{3}\right)_{2}, 1.25 \mathrm{mM} \mathrm{KNO}{ }_{3}\right.$, $0.5 \mathrm{mM} \mathrm{MgSO}, 0.25 \mathrm{mM} \mathrm{KH_{2 }} \mathrm{PO}_{4}$; micronutrients: $10 \mu \mathrm{M} \mathrm{H}_{3} \mathrm{BO}_{3}, 1 \mu \mathrm{M} \mathrm{MnSO}_{4}, 0.5 \mu \mathrm{M} \mathrm{ZnSO}_{4}, 0.05 \mu \mathrm{M}$
$\left(\mathrm{NH}_{4}\right)_{6} \mathrm{Mo}_{7} \mathrm{O}_{24}$ and $\left.0.4 \mu \mathrm{M} \mathrm{CuSO}_{4}\right)$ following Jelali et al. (2010). The nutrient solution was aerated with the aid of aquarium diffusers. One week later, seedlings were transferred to $37 \mathrm{~L}$ boxes (15 plants per box) filled with the same nutrient solution that was replaced weekly. Growing conditions in the growth chamber were $16 \mathrm{~h} \mathrm{light} / 8 \mathrm{~h}$ dark photoperiod, $18 / 20{ }^{\circ} \mathrm{C}, 65 \%$ humidity, and a photon flux density of $200-300 \mu \mathrm{M} \mathrm{m}^{-2} \mathrm{~s}^{-1}$. Plants used in the experiments were six- to seven-week old.

\section{RNA extraction and PsPIP genes identification}

Total RNA was extracted from roots and leaves of Pea plants using Trizol regent (Invitrogen, Grand Island, NY, USA) following the manufacturer's instructions. The concentration of RNA was quantified by spectrophotometrical measurement at $\lambda=260 \mathrm{~nm}$, and its integrity was checked on agarose gels. First strand cDNA was synthesized from $2 \mu \mathrm{g}$ of total RNA using GoScript reverse transcription regent Kit (Promega, Madison, WI, USA). The synthesized cDNA was amplified by polymerase chain reaction (PCR) using oligo(dT) and degenerate oligonucleotide primers (Additional file 1: Table S1) which were designed from the known sequences of different plant PIP genes. The PCR products were gel-purified and sub-cloned into pMD18-T vector (Takara, TAKARA Biotechnology Co. Ltd, Dalian, China), and the constructed plasmids were transformed into E. coli $\mathrm{DH} 5 \alpha$. The positive clones were sequenced and analyzed. Next, $5^{\prime}$-rapid amplification of cDNA ends (RACE) was applied to clone the $5^{\prime}$-end sequences of the PIP genes. Sequences analyses with database were performed at NCBI (http://www. ncbi.nlm.nih.gov/) using the BLAST network services, and a phylogenetic tree was generated in MEG5.1 software (http://www.megasoftware.net) to test the evolutionary relationships.

\section{The Silencing of PsPIP2; 1 in Pea plants}

To optimize the VIGS method, Constantin et al. (2004) transferred the RNA1 and RNA2 expression cassettes of a Pea early browning virus (PEBV) to the binary agrobacterium vector pCAMBIA1300. Then, pCAMBIA1300derived plasmid with the expression cassette of RNA1 was named as PCAPE1, and pCAMBIA1300-derived plasmid with the expression cassette of RNA2-GFP was named as pCAPE2-GFP. In the present study, sequence of GFP in pCAPE2-GFP was replaced with cDNA fragment of $P$. sativum phytoene desaturase (PDS) gene and with partial encoding region of PsPIP2;1 plus $3^{\prime} \mathrm{UTR}$ sequence to obtain pCAPE2-PDS and pCAPE2-PsPIP2;1, respectively. Also, a vector control plasmid, pCAPE2Con was constructed by replacing the GFP sequence of pCAPE2-GFP with a fragment derived from the cDNA of Bean yellow mosaic virus (AJ622899). Next, the 


\section{a Roots}

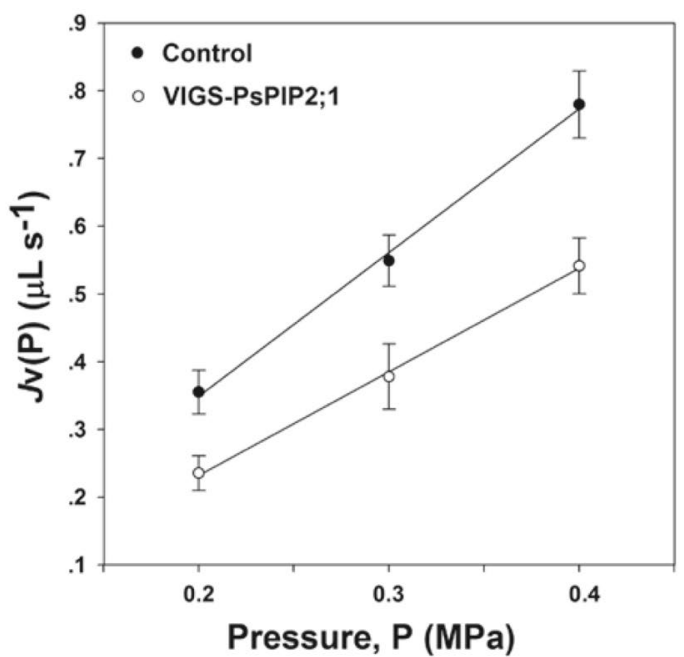

b Roots

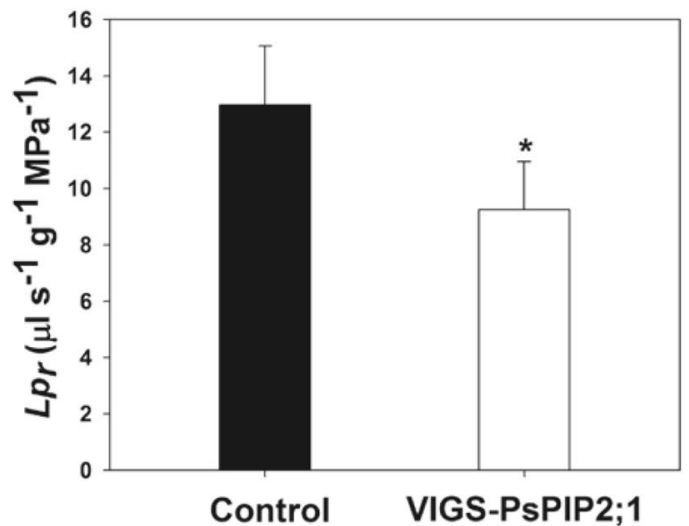

\section{Root cells}

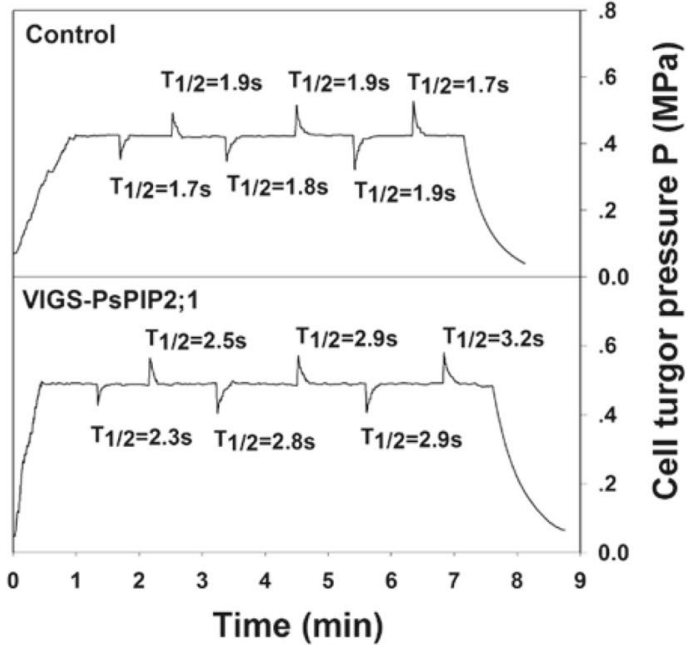

\section{d Root cells}

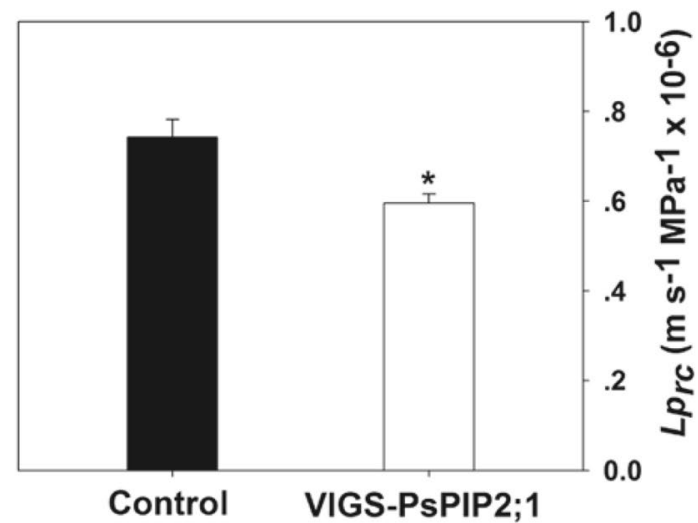

Fig. 4 Virus induced PSPIP2;1 silencing led to reductions in root hydraulic conductivities. a Representative pressure-to-flow relationship measured in roots of control plants and VIGS-PSPIP2;1 plants. Jv represents the rate of exuded sap flow through roots. b Root hydraulic conductivity at organ level. c Typical hydrostatic relaxation curves as measured by a cell pressure probe on root cells. $\mathbf{d}$ Root hydraulic conductivity at cell level. Values are mean \pm SD ( $n=6-9$ plants or $30-60$ cells). Asterisks $\left(^{*}\right)$ represent a significant difference between control plants and VIGS-PsPIP2; 1 plants at $P<0.05$

constructed plasmids including pCAPE1, pCAPE2-PDS, pCAPE2-PsPIP2;1, and pCAPE2-Con were transformed separately into Agrobacterium tumefaciens GV3101 using the freeze-thaw method (Hofgen and Willmitzer 1988). Two-week old Pea plants were infiltrated at the abaxial side of the youngest pair of leaves with agrobacterium cultures carrying pCAPE1 and the pCAPE2-derived plasmids at a 1:1 ratio. Plants were separated into three groups that were subsequently inoculated with three different agrobacterium cultures: (1) pCAPE2-PDS, which served as an indicator of gene silencing, in that PDS silenced plants had photo-bleached leaves (as a result of lacking carotenoids and destruction of chlorophyll by photo-oxidation), and this phenotype was associated with a significant reduction in PsPDS mRNA (Kumagai et al. 1995); (2) pCAPE2-PsPIP2;1 to silence the target PSPIP2;1 gene; and (3) pCAPE2-Con as the control. When the target gene was silenced, as indicated by the photo-bleached leaves of PDS silenced plants, the shoots of PSPIP2;1 silenced plants were labeled at the position where the photo-bleached phenotype began to appear (Additional file 2: Figure S1). Meanwhile, roots of PSPIP2;1 silenced plants were cut back to approximately $3 \mathrm{~cm}$ and root growth was allowed to re-initiate. Then plants were grown in the growth chamber for additional 2-3 weeks to allow the production of newly emerged leaves and regenerative roots, which were used in subsequent experiments. 
Table 1 Cell pressure probe measurements of root cortex cells and leaf epidermal cells of control plants and virus induced PsPIP2; 1 silencing plants (VIGS-PsPIP2;1)

\begin{tabular}{lcc}
\hline & Control & VIGS-PsPIP2; 1 \\
\hline Root cortical cell & & \\
Turgor pressure, $\mathrm{P}(\mathrm{MPa})$ & $0.38 \pm 0.08 \mathrm{a}$ & $0.37 \pm 0.06 \mathrm{a}$ \\
Cell volume, $\mathrm{V}\left(\mathrm{m}^{3}\right)$ & $1.7 \pm 0.3 \mathrm{E}-13 \mathrm{a}$ & $1.6 \pm 0.2 \mathrm{E}-13 \mathrm{a}$ \\
Cell surface area, $\mathrm{A}\left(\mathrm{m}^{2}\right)$ & $2.2 \pm 0.4 \mathrm{E}-08 \mathrm{a}$ & $2.1 \pm 0.3 \mathrm{E}-08 \mathrm{a}$ \\
$\varepsilon(\mathrm{MPa})$ & $4.0 \pm 1.0 \mathrm{a}$ & $3.9 \pm 1.2 \mathrm{a}$ \\
$\mathrm{T}_{1 / 2}(\mathrm{~s})$ & $1.8 \pm 0.1 \mathrm{a}$ & $2.6 \pm 0.6 \mathrm{~b}$ \\
Leaf epidermal cell & & \\
Turgor pressure, $\mathrm{P}(\mathrm{MPa})$ & $0.34 \pm 0.05 \mathrm{a}$ & $0.32 \pm 0.08 \mathrm{a}$ \\
Cell volume, $\mathrm{V}\left(\mathrm{m}^{3}\right)$ & $1.1 \pm 0.2 \mathrm{E}-13 \mathrm{a}$ & $1.2 \pm 0.1 \mathrm{E}-13 \mathrm{a}$ \\
Cell surface area, $\mathrm{A}\left(\mathrm{m}^{2}\right)$ & $1.7 \pm 0.5 \mathrm{E}-08 \mathrm{a}$ & $1.6 \pm 0.4 \mathrm{E}-08 \mathrm{a}$ \\
$\varepsilon(\mathrm{MPa})$ & $2.2 \pm 1.1 \mathrm{a}$ & $2.3 \pm 1.2 \mathrm{a}$ \\
$\mathrm{T}_{1 / 2}(\mathrm{~s})$ & $1.2 \pm 0.2 \mathrm{a}$ & $1.7 \pm 0.4 \mathrm{~b}$ \\
\hline
\end{tabular}

Results are presented as mean $\pm S D(n=30-60$ cells). Different letters indicate significant differences $(P<0.05)$

\section{Quantitative real-time PCR (q-RT-PCR) analyses}

Total RNA extraction, concentration and integrity were determined as described above. First strand cDNA was synthesized using primeScript RT regent Kit (TakaRa, TAKARA Biotechnology Co. Ltd, Dalian, China) following manufacturer's instructions, including a special step for genomic DNA elimination. Quantitative PCR analysis was conducted on an ABI 7500 Real-Time system using a SYBR Green Premix Ex-Taq ${ }^{\mathrm{TM}}$ II Kit (TakaRa, TAKARA Biotechnology Co. Ltd, Dalian, China) with PsPIP gene specific primers (Additional file 3: Table S2). The reaction mixture had a final volume of $20 \mu \mathrm{L}$, containing $10 \mu \mathrm{L} 2 \times$ SYBR Premix Ex Taq ${ }^{\mathrm{TM}}$ II, $0.4 \mu \mathrm{M}$ of each primer, $0.4 \mu \mathrm{L} 50 \times$ ROX Reference Dye II and $2 \mu \mathrm{L}$ of tenfold dilution cDNA. The PCR conditions were: $30 \mathrm{~s}$ at $95{ }^{\circ} \mathrm{C}$ for pre-denaturation; 40 cycles of $5 \mathrm{~s}$ at $95^{\circ} \mathrm{C}, 34 \mathrm{~s}$ at $60{ }^{\circ} \mathrm{C}$. The melt-curve analysis was conducted using the method recommended by the manufacturer. The results were normalized by the geometric mean of the expression of three reference genes, i.e., elongation factor 1-alpha (EF1 $\alpha, \mathrm{X} 96555), 18 \mathrm{~s}$ ribosomal RNA (18 s, X52575) and beta-tubulin 3 (TUB, X54846). The relative expression of PsPIPs was calculated using the $2^{-\Delta \Delta \mathrm{Ct}}$ method (Pfaffl 2001; Schmittgen and Livak 2008).

\section{Root and leaf hydraulic conductivity measurements}

Root and leaf hydraulic conductivity $\left(\operatorname{Lp}_{\mathrm{r}}\right.$ and $K_{\text {leaf }}$ respectively) was measured using the pressure chamber technique following Javot et al. (2003) and Postaire et al. (2010), with slight modifications. For $\mathrm{Lp}_{\mathrm{r}}$ measurements, shoots were cut off below the first node of the plants, and the whole roots were bathed in nutrient solution in a pressure chamber (PMS, Corvallis, OR, USA). The hypocotyl was carefully threaded through the soft plastic washer of the metal lid. Pressure $(\mathrm{P})$ that was generated by compressed air in steps of $0.1 \mathrm{MPa}$ (up to $0.5 \mathrm{MPa}$ ) was slowly applied to the chamber, and the rate of exuded sap flow $\left(J_{\mathrm{v}}\right)$ was determined. When $J_{\mathrm{v}}$ was plotted against the applied $\mathrm{P}$, a linear relationship was observed for $\mathrm{P}$ values between 0.2 and $0.4 \mathrm{MPa}$ (Fig. 4a). At the end of the measurement, the root system was removed and dry weight (DW) of the roots (after oven-dried at $70{ }^{\circ} \mathrm{C}$ for $72 \mathrm{~h}$ ) was measured using a balance (FA2104N, Minqiao Instrument Co. Ltd, Shanghai, China). Lp $\left(\mu \mathrm{L} \mathrm{s}^{-1} \mathrm{~g}^{-1} \mathrm{MPa}^{-1}\right)$ was calculated from the slope of the exuded sap flow rate versus pressure, divided by DW of the roots.

Similarly, for $K_{\text {leaf }}$ determination, a detached mature compound leaf was inserted into a pressure chamber (PMS, Corvallis, OR, USA) filled with distilled water. The common petiole was carefully threaded through the soft plastic washer of the metal lid. Pressure was applied to the chamber in steps of $0.1 \mathrm{MPa}$ (up to $0.5 \mathrm{MPa}$ ), using compressed air gas. This resulted in a flow of liquid $\left(J_{\mathrm{v}}\right)$ entering through the leaf surface and exiting from the common petiole. When $J_{\mathrm{v}}$ was plotted against $\mathrm{P}$, a linear relationship was observed for $\mathrm{P}$ values between 0.3 and $0.5 \mathrm{MPa}$ (Fig. 5a). At the end of the measurement, leaves were scanned and the surface area $(S)$ was measured using Image J software v1.42 (Bethesda, MD, USA). $K_{\text {leaf }}$ $\left(\mu \mathrm{L} \mathrm{s}^{-1} \mathrm{~m}^{-2} \mathrm{MPa}^{-1}\right)$ was calculated from the slope of the exuded sap flow rate versus pressure, divided by $S$ of the leaves.

\section{Cell pressure probe measurements}

Cell pressure probe (CPP) measurements were performed as described in Steudle (1993). In brief, pulled glass micro-capillary were beveled to a tip diameter of 5-7 $\mu \mathrm{m}$, filled with silicone oil (type AS4; Wacker, Munich, Germany), and mounted to the CPP. To measure root cell hydraulic conductivity $\left(\mathrm{Lp}_{\mathrm{rc}}\right)$, root segment from plants grown in hydroponic condition was fixed by magnetic bars on a metal sledge which was covered with wet filter paper. An aerated nutrient solution was circulated along the root segment to maintain moisture. Root cells were punctured using a CPP, and cell sap entered the oil-filled micro-capillary forming a meniscus between cell sap and oil. Cell turgor pressure was restored by gently pushing the meniscus to a position close to the surface of the root, and the values of cell turgor pressure (P) were recorded by a computer (Ye et al. 2004). Half time of water exchange $\left(T_{1 / 2}\right)$ across cell membranes was obtained from hydrostatic pressure relaxation curves with the aid of the probe. After CPP measurements, average values of cell volume and surface area were obtained 


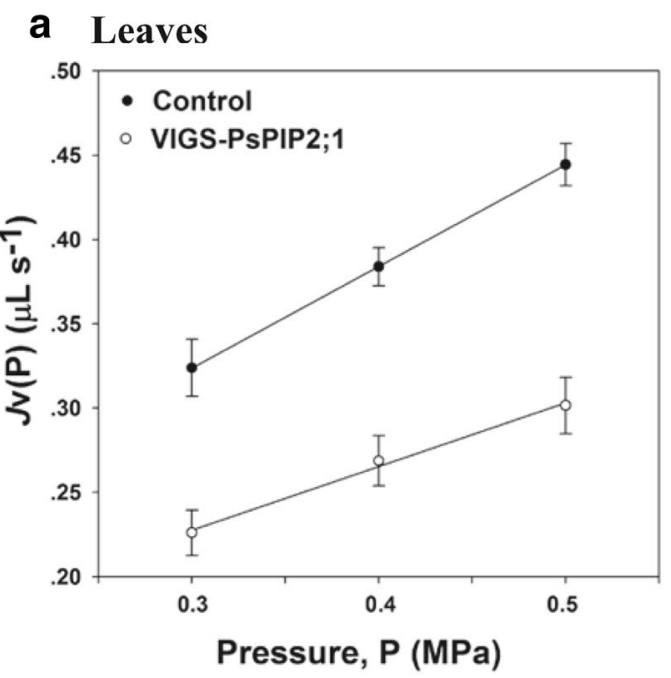

b Leaves

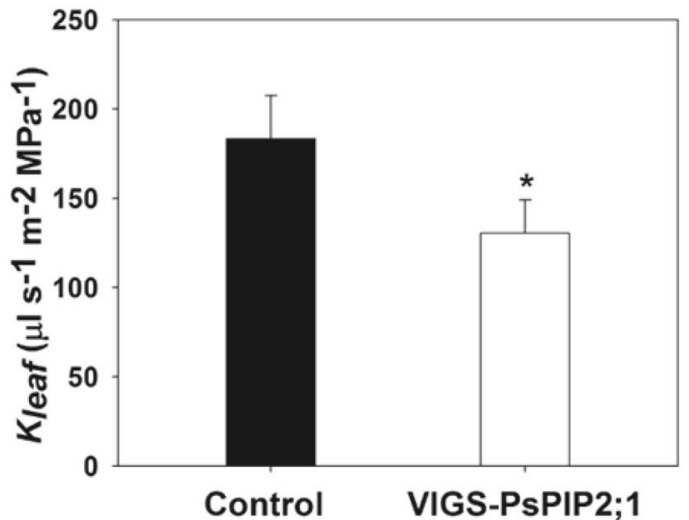

C Leaf cells

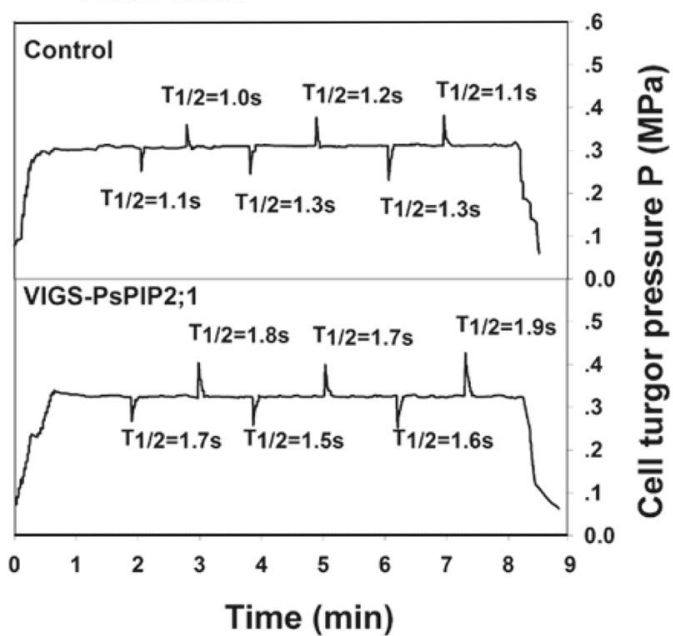

\section{d Leaf cells}

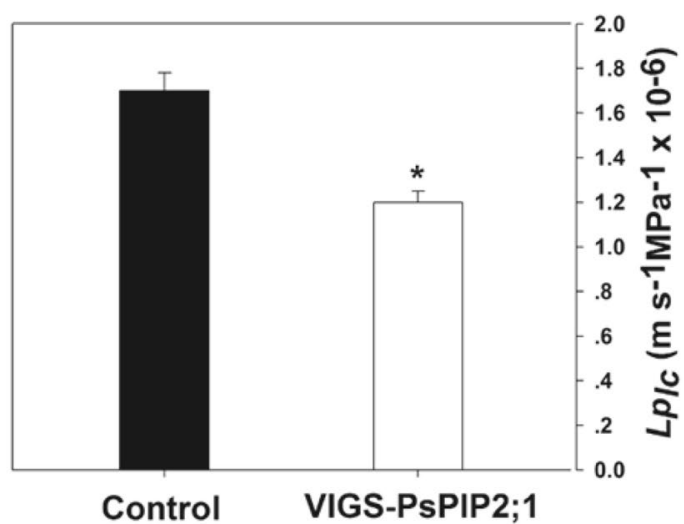

Fig. 5 Virus induced PSPIP2; 1 silencing led to reductions in leaf hydraulic conductivities. a Representative pressure-to-flow relationship measured in leaves of control plants and VIGS-PSPIP2;1 plants. $J_{V}$ represents the rate of exuded sap flow through leaves. $\mathbf{b}$ Leaf hydraulic conductivity at organ level. c Typical hydrostatic relaxation curves as measured by a cell pressure probe on leaf cells. $\mathbf{d}$ Leaf hydraulic conductivity at the cell level. Values are mean $\pm S D\left(n=6-9\right.$ plants or 30-60 cells). Asterisks $\left(^{*}\right)$ represent a significant difference between control plants and VIGS-PSPIP2;1 plants at $P<0.05$

through microscopic analyses with root sections, assuming that cells had a cylindrical shape. $\mathrm{Lp}_{\mathrm{rc}}$ was calculated according to the following equation:

$$
\mathrm{Lp}=\frac{V}{A} \times \frac{\ln (2)}{T_{1 / 2}^{w}\left(\varepsilon+\pi^{i}\right)}
$$

Here, $\mathrm{V}=$ cell volume; $\mathrm{A}=$ cell surface area; $\pi^{\mathrm{i}}=$ osmotic pressure of cell sap; $\varepsilon=$ cell elastic modulus. $\pi^{\mathrm{i}}$ was calculated from the initial cell turgor $\left(\mathrm{P}_{0}\right)$, as $P_{0}=\pi^{i}-\pi^{0}\left(\pi^{0}=\right.$ osmotic pressure of the medium measured with an osmometer); elastic modulus was determined from relative change of cell volume $(\Delta \mathrm{V} / \mathrm{V})$ and the instantaneous change of cell turgor $(\Delta \mathrm{P})$ :

$$
\varepsilon=V \times \frac{\Delta P}{\Delta V}
$$

Where the change in cell volume $(\Delta \mathrm{V})$ was induced by moving the meniscus with the aid of the CPP, which was calculated from the length of meniscus movement in the micro-capillary using the eyepiece reticule of the microscope under a given magnification, and from the inner diameter of the capillary where the meniscus located (Steudle 1993).

For leaf cell hydraulic conductivity $\left(\mathrm{Lp}_{\mathrm{lc}}\right)$ measurements, a mature young leaf blade (still attached to the plant) was fixed onto a metal sledge. Leaf cells were punctured using a CPP, and water relation parameters such as 
$\mathrm{T}_{1 / 2}$, $\varepsilon$, and $\mathrm{Lp}_{\mathrm{Ic}}$ were determined as described above for root cell measurements.

\section{Statistical analysis}

Results were presented as mean \pm SD of three independent experiments. Statistical analyses were performed using SPSS 13.0 program (Chicago, IL, USA). Statistical significant differences were determined by $t$ test at $\mathrm{P}<0.05$.

\section{Additional files}

Additional file 1: Table S1. Sequences of degenerate oligo nucleotide primers designed from the known sequences of different plant PIP genes.

Additional file 2: Table S2. Sequences of gene-specific primers used for real-time RT-PCR amplification.

Additional file 3: Figure S1. Virus-induced gene silencing of $P$. sativum phytoene desaturase (PSPDS). (A) Leaves of a control plant inoculated with PEBV carrying a fragment of Bean yellow mosaic virus (pCAPE2Con) remained green; (B) leaves of a plant inoculated with PEBV carrying a fragment of PSPDS (pCAPE2-PDS) showed a characteristic bleaching phenotype.

\section{Abbreviations}

AQPs: aquaporins; CPP: cell pressure probe; $K_{\text {leaf }}$ leaf hydraulic conductivity; Lp: : leaf cell hydraulic conductivity; Lp: root hydraulic conductivity; LP $p_{r}$ : root cell hydraulic conductivity; PIP: plasma membrane intrinsic protein; q-RT-PCR: quantitative real-time PCR; $T_{1 / 2}$ : half-time of water exchange; VIGS: virusinduced gene silencing.

\section{Authors' contributions}

JS and QY conceived and designed the project. JS, GY and ZQ conducted the experiments, JS and QY analyzed the results. All authors contributed to the writing of the manuscript. All authors read and approved the final manuscript.

\section{Author details}

${ }^{1}$ Key Laboratory of Vegetation Restoration and Management of Degraded Ecosystems, South China Botanical Garden, Chinese Academy of Sciences, 723 Xingke Road, Tianhe District, Guangzhou 510650, China. ${ }^{2}$ Guangdong Provincial Key Laboratory of Applied Botany, South China Botanical Garden, Chinese Academy of Sciences, 723 Xingke Road, Tianhe District, Guangzhou 510650, Guangdong, China. ${ }^{3}$ University of Chinese Academy of Sciences, 19A Yuquan Road, Beijing 100049, China.

\section{Acknowledgements}

We are grateful to two anonymous Reviewers for their insightful comments and constructive suggestions on an earlier version of this article. We thank Dr. J Yang (Institute of Plant Physiology and Ecology, Chinese Academy of Sciences, Shanghai, China) for providing seeds of P. sativum $L$. This work was funded by Natural Science Foundation of Guangdong Province, China (2014A030313787), the National Natural Science Foundation of China (31070231), and the Scientific Research Foundation for the Returned Overseas Chinese Scholars, State Education Ministry of China.

\section{Competing interests}

The authors declare that they have no competing interests.

\section{Availability of supporting data}

All supporting data are included as additional files.

Received: 2 March 2016 Accepted: 13 July 2016

Published online: 06 August 2016

\section{References}

Aharon R, Shahak Y, Wininger S, Bendov R, Kapulnik Y, Galili G (2003) Overexpression of a plasma membrane aquaporin in transgenic tobacco improves plant vigor under favorable growth conditions but not under drought or salt stress. Plant Cell 15:439-447

Anderberg HI, Danielson JAH, Johanson U (2011) Algal MIPs, high diversity and conserved motifs. BMC Evol Biol 11:1. doi:10.1186/1471-2148-11-110

Aroca R, Porcel R, Manuel Ruiz-Lozano J (2012) Regulation of root water uptake under abiotic stress conditions. J Exp Bot 63:43-57

Ayadi M, Cavez D, Miled N, Chaumont F, Masmoudi K (2011) Identification and characterization of two plasma membrane aquaporins in durum wheat (Triticum turgidum L. subsp. durum) and their role in abiotic stress tolerance. Plant Physiol Biochem 49:1029-1039

Baulcombe DC (1999) Fast forward genetics based on virus-induced gene silencing. Curr Opin Plant Biol 2:109-113

Beaudette PC, Chlup M, Yee J, Emery RJN (2007) Relationships of root conductivity and aquaporin gene expression in Pisum sativum: diurnal patterns and the response to $\mathrm{HgCl}_{2}$ and ABA. J Exp Bot 58:1291-1300

Bramley H, Turner NC, Turner DW, Tyerman SD (2009) Roles of morphology, anatomy, and aquaporins in determining contrasting hydraulic behavior of roots. Plant Physiol 150:348-364

Burch-Smith TM, Anderson JC, Martin GB, Dinesh-Kumar SP (2004) Applications and advantages of virus-induced gene silencing for gene function studies in plants. Plant J 39:734-746

Chaumont F, Tyerman SD (2014) Aquaporins: highly regulated channels controlling plant water relations. Plant Physiol 164:1600-1618

Chaumont F, Barrieu F, Jung R, Chrispeels MJ (2000) Plasma membrane intrinsic proteins from maize cluster in two sequence subgroups with differential aquaporin activity. Plant Physiol 122:1025-1034

Chevalier AS, Chaumont F (2015) Trafficking of plant plasma membrane aquaporins: multiple regulation levels and complex sorting signals. Plant Cell Physiol 56:819-829

Cochard $\mathrm{H}$ et al (2007) Putative role of aquaporins in variable hydraulic conductance of leaves in response to light. Plant Physiol 143:122-133

Constantin GD, Krath BN, MacFarlane SA, Nicolaisen M, Johansen IE, Lund OS (2004) Virus-induced gene silencing as a tool for functional genomics in a legume species. Plant J 40:622-631

Da Ines $O$ et al (2010) Kinetic analyses of plant water relocation using deuterium as tracer-reduced water flux of Arabidopsis pip2 aquaporin knockout mutants. Plant Biol 12:129-139

Ding X, Iwasaki I, Kitagawa Y (2004) Overexpression of a lily PIP1 gene in tobacco increased the osmotic water permeability of leaf cells. Plant Cell Environ 27:177-186

Fragkostefanakis S, Sedeek KEM, Raad M, Zaki MS, Kalaitzis P (2014) Virus induced gene silencing of three putative prolyl 4-hydroxylases enhances plant growth in tomato (Solanum lycopersicum). Plant Mol Biol 85:459-471

Gustavsson S, Lebrun AS, Norden K, Chaumont F, Johanson U (2005) A novel plant major intrinsic protein in Physcomitrella patens most similar to bacterial glycerol channels. Plant Physiol 139:287-295

Heinen RB, Ye Q, Chaumont F (2009) Role of aquaporins in leaf physiology. J Exp Bot 60:2971-2985

Henry A, Cal AJ, Batoto TC, Torres RO, Serraj R (2012) Root attributes affecting water uptake of rice (Oryza sativa) under drought. J Exp Bot 63:4751-4763

Hofgen R, Willmitzer L (1988) Storage of competent cells for Agrobacterium transformation. Nucleic Acids Res 16:9877

Jang JY, Lee SH, Rhee JY, Chung GC, Ahn SJ, Kang H (2007) Transgenic Arabidopsis and tobacco plants overexpressing an aquaporin respond differently to various abiotic stresses. Plant Mol Biol 64:621-632

Javot H, Maurel C (2002) The role of aquaporins in root water uptake. Ann Bot 90:301-313

Javot $\mathrm{H}$ et al (2003) Role of a single aquaporin isoform in root water uptake. Plant Cell 15:509-522

Jelali N, Dell'Orto M, Rabhi M, Zocchi G, Abdelly C, Gharsalli M (2010) Physiological and biochemical responses for two cultivars of Pisum sativum ("Merveille de Kelvedon" and "Lincoln") to iron deficiency conditions. Sci Hortic 124:116-121 
Johanson U et al (2001) The complete set of genes encoding major intrinsic proteins in Arabidopsis provides a framework for a new nomenclature for major intrinsic proteins in plants. Plant Physiol 126:1358-1369

Johansson I, Karlsson M, Johanson U, Larsson C, Kjellbom P (2000) The role of aquaporins in cellular and whole plant water balance. Biochim Biophys Acta-Biomembr 1465:324-342

Kaneko T, Horie T, Nakahara Y, Tsuji N, Shibasaka M, Katsuhara M (2015) Dynamic regulation of the root hydraulic conductivity of barley plants in response to salinity/osmotic stress. Plant Cell Physiol 56:875-882

Kim YMX, Steudle E (2007) Light and turgor affect the water permeability (aquaporins) of parenchyma cells in the midrib of leaves of Zea mays. J Exp Bot 58:4119-4129

Kumagai MH, Donson J, Dellacioppa G, Harvey D, Hanley K, Grill LK (1995) Cytoplasmic inhibition of carotenoid biosynthesis with virus-derived RNA. Proc Natl Acad Sci USA 92:1679-1683

Lee SH, Chung GC, Jang JY, Ahn SJ, Zwiazek JJ (2012) Overexpression of PIP2;5 aquaporin alleviates effects of low root temperature on cell hydraulic conductivity and growth in Arabidopsis. Plant Physiol 159:479-488

Li G, Santoni V, Maurel C (2014) Plant aquaporins: roles in plant physiology. Biochim Biophys Acta-Gen Subj 1840:1574-1582

Lopez-Berenguer C, Martinez-Ballesta MC, Garcia-Viguera C, Carvajal M (2008) Leaf water balance mediated by aquaporins under salt stress and associated glucosinolate synthesis in broccoli. Plant Sci 174:321-328

Lu R, Martin-Hernandez AM, Peart JR, Malcuit I, Baulcombe DC (2003) Virusinduced gene silencing in plants. Methods 30:296-303

Luu DT, Maurel C (2005) Aquaporins in a challenging environment: molecular gears for adjusting plant water status. Plant, Cell Environ 28:85-96

Maggio A, Joly RJ (1995) Effects of mercuric-chloride on the hydraulic conductivity of tomato root systems - evidence for a channel-mediated water pathway. Plant Physiol 109:331-335

Martre P, Morillon R, Barrieu F, North GB, Nobel PS, Chrispeels MJ (2002) Plasma membrane aquaporins play a significant role during recovery from water deficit. Plant Physiol 130:2101-2110

Maurel C, Verdoucq L, Luu D-T, Santoni V (2008) Plant aquaporins: membrane channels with multiple integrated functions. In: Merchant SS (ed) Annual Review of Plant Biology. Annual reviews, California. 59, p 595-624

Muries B, Carvajal M, del Carmen Martinez-Ballesta M (2013) Response of three broccoli cultivars to salt stress, in relation to water status and expression of two leaf aquaporins. Planta 237:1297-1310

Perrone I et al (2012) The grapevine root-specific aquaporin VVPIP2:4N controls root hydraulic conductance and leaf gas exchange under well-watered conditions but not under water stress. Plant Physiol 160:965-977

Pfaffl MW (2001) A new mathematical model for relative quantification in realtime RT-PCR. Nucleic Acids Res 29:2002-2007
Pflieger S, Richard MMS, Blanchet S, Meziadi C, Geffroy V (2013) VIGS technology: an attractive tool for functional genomics studies in legumes. Funct Plant Biol 40:1234-1248

Postaire O, Tournaire-Roux C, Grondin A, Boursiac Y, Morillon R, Schaeffner AR, Maurel C (2010) A PIP1 aquaporin contributes to hydrostatic pressureinduced water transport in both the root and rosette of Arabidopsis. Plant Physiol 152:1418-1430

Prado K et al (2013) Regulation of Arabidopsis leaf hydraulics involves light-dependent phosphorylation of aquaporins in veins. Plant Cell 25:1029-1039

Purkayastha A, Dasgupta I (2009) Virus-induced gene silencing: a versatile tool for discovery of gene functions in plants. Plant Physiol Biochem 47:967-976

Purkayastha A, Mathur S, Verma V, Sharma S, Dasgupta I (2010) Virus-induced gene silencing in rice using a vector derived from a DNA virus. Planta 232:1531-1540

Qian Z-J, Song J-J, Chaumont F, Ye Q (2015) Differential responses of plasma membrane aquaporins in mediating water transport of cucumber seedlings under osmotic and salt stresses. Plant, Cell Environ 38:461-473

Schmittgen TD, Livak KJ (2008) Analyzing real-time PCR data by the comparative C-T method. Nat Protoc 3:1101-1108

Schuurmans J, van Dongen JT, Rutjens BPW, Boonman A, Pieterse CMJ, Borstlap AC (2003) Members of the aquaporin family in the developing pea seed coat include representatives of the PIP, TIP, and NIP subfamilies. Plant Mol Biol 53:655-667

Secchi F, Zwieniecki MA (2014) Down-regulation of plasma intrinsic protein1 aquaporin in poplar trees is detrimental to recovery from embolism. Plant Physiol 164:1789-1799

Senthil-Kumar M, Mysore KS (2014) Tobacco rattle virus-based virus-induced gene silencing in Nicotiana benthamiana. Nat Protoc 9:1549-1562

Steudle E (1993) Pressure probe techniques: basic principles and application to studies of water and solute relations at the cell, tissue and organ level. In: Smith JAC, Griffiths H (eds) Water deficits: plant responses from cell to community. Bios Scientific Publishers, Oxford, pp 5-36

Ye Q, Wiera B, Steudle E (2004) A cohesion/tension mechanism explains the gating of water channels (aquaporins) in Chara internodes by high concentration. J Exp Bot 55:449-461

Yu QJ, Hu YL, Li JF, Wu Q, Lin ZP (2005) Sense and antisense expression of plasma membrane aquaporin BnPIP1 from Brassica napus in tobacco and its effects on plant drought resistance. Plant Sci 169:647-656

Zhang WH, Tyerman SD (1999) Inhibition of water channels by $\mathrm{HgCl}_{2}$ in intact wheat root cells. Plant Physiol 120:849-857

\section{Submit your manuscript to a SpringerOpen ${ }^{\circ}$ journal and benefit from:}

- Convenient online submission

- Rigorous peer review

- Immediate publication on acceptance

- Open access: articles freely available online

High visibility within the field

- Retaining the copyright to your article

Submit your next manuscript at springeropen.com 